\title{
Ultrahigh Strength and Shear-Assisted Decohesion of Sliding Silver Nanocontacts Studied in situ
}

\author{
Takaaki Sato ( $\nabla$ takaaki19821212ab@gmail.com ) \\ University of Pennsylvania https://orcid.org/0000-0002-8826-0936
}

\section{Zachary Milne}

Sandia National Laboratories

\section{Masahiro Nomura}

University of Tokyo https://orcid.org/0000-0003-3706-4836

\section{Naruo Sasaki}

The University of Electro-Communications

\section{Robert Carpick}

University of Pennsylvania

\section{Hiroyuki Fujita}

University of Tokyo

\section{Article}

Keywords: nanoscale adhesion, nanoscale friction, nanotribology, surface energy, cold welding, high resolu-tion transmission electron microscopy, nanoindentation, in situ, fracture, silver, grain boundary, ideal strength, cohesive fracture

Posted Date: September 23rd, 2021

DOI: https://doi.org/10.21203/rs.3.rs-798463/v1

License: (c) (1) This work is licensed under a Creative Commons Attribution 4.0 International License. Read Full License

Version of Record: A version of this preprint was published at Nature Communications on May 10th, 2022. See the published version at https://doi.org/10.1038/s41467-022-30290-y. 


\section{Ultrahigh Strength and Shear-Assisted Decohesion of Sliding Silver Nano- contacts Studied in situ}

Takaaki Sato ${ }^{1}$, Zachary B. Milne ${ }^{2}$, Masahiro Nomura ${ }^{3}$, Naruo Sasaki ${ }^{4}$, Robert W. Carpick ${ }^{1}$, Hiroyuki Fujita $^{3,5}$

1 University of Pennsylvania, Department of Mechanical Engineering and Applied Mechanics, Philadelphia, PA, USA

2 Sandia National Laboratories, Nanostructure Physics, Albuquerque, NM, USA

3 University of Tokyo, Institute of Industrial Science. Tokyo, JP.

4 The University of Electro-Communications, Department of Engineering Science, Tokyo, JP

5 Tokyo city university, Graduate school of integrative science and engineering electrical and electronic engineering, Tokyo, JP

Primary contact email: takaakis@ seas.upenn.edu phone: +81-3-5452-6249 fax: +81-3-5452-6250

\section{Abstract}

The fundamental behavior of materials in sliding contact is challenging to determine since the interface is normally hidden from view. Using a custom microfabricated device, we conducted in situ, ultrahigh vacuum transmission electron microscope measurements of silver-silver crystalline nanocontacts under combined tension and shear, permitting simultaneous observation of contact forces and contact width. Here, we find the Ag-Ag contacts exhibit only limited shear-induced junction growth, unlike the larger growth seen at macroscales. The difference arises from the nanocontacts' high strength. The von Mises stresses at yield points approach the ideal strength of silver, attributed to the nearly defect-free nature and small size. The results are consistent with recent predictions that nanocrystalline fec metals yield via interfacial amorphization. The contacts separate unstably, with pull-off forces well below predictions for rupture under pure tension. This provides in situ evidence that shear reduces nanoscale pull-off forces, consistent with theoretical predictions for this effect.

Keywords: nanoscale adhesion, nanoscale friction, nanotribology, surface energy, cold welding, high resolution transmission electron microscopy, nanoindentation, in situ, fracture, silver, grain boundary, ideal strength, cohesive fracture 
Understanding and predicting the behavior of materials in sliding contact is highly challenging, particularly because of the breadth of physical phenomena that can occur, all at a confined interface normally hidden from direct view [1,2]. For metal-metal interfaces, the presence of strong adhesion (once contaminants and/or oxides are removed, which can readily occur in sliding contacts) combined with any applied load can often lead to ductile flow, strain hardening, and cold welding [3,4]. Unraveling and understanding these can help develop better models for describing and predicting the behavior of practical engineering interfaces, which will consist of multitudes of such asperity contacts due to the typical roughness of surfaces. Metal-metal contact often occurs in machinery and engines, and is important in a wide range of fields including transportation, power conversion, manufacturing, medical device implants, and microand and nanoelectromechanical system (MEMS/NEMS).

Extensive studies have used atomic force microscopy (AFM) and related techniques to explore single-asperity nanotribology, with the advantage that single asperity behavior can be measured with nanoscale force and displacement resolution and provide a well-defined contact geometry $[5,6]$ While the contact is not directly observed in standard AFM systems, intermittent characterization of the tip size and shape, and indirect measurements of physical quantities related to contact size such as contact stiffness, contact conductance, and adhesion provide valuable information on the contact geometry $[5,6]$.

While the behavior of nanoscale metallic junctions subjected to normal loading has been studied by AFM or AFM-based methods, e.g., [7-10], fewer studies have probed metal-metal nanocontacts in frictional sliding. In one example, a probe was traced across a metal surface such as $\mathrm{Au}$ and $\mathrm{Cu}$ with a load of less than $1 \mathrm{nN}$ [11]. Stick-slip instabilities in the frictional force with atomic regularity were observed, but with extremely low dissipation when low loads were used. This was attributed to low shear of (111) planes in a metallic neck that the authors suggest was formed between the $\mathrm{Cu}(100)$ sample and the Si tip. At higher loads, irregular stick-slip and higher friction occurred, similar to previous measurements on $\mathrm{Cu}(100)$ surfaces [12]. 
Directly observing the shape and size of the contact could elucidate the extent of elastic deformation and the degree of agreement at the nanoscale of continuum contact models such as the Johnson-KendallRoberts (JKR) [13] or the Derjaguin-Müller-Toporov (DMT) [14] models, whose validity at this scale is an active research topic $[15,16]$. Observing inelastic behavior, such as dislocation activity and plastic flow, material transfer, and cold welding would enable determination of the deformation regime of the materials for robust models for asperity-level contact. Such observations would also permit observing the nanoscale origins of important phenomena known or inferred in macroscale contacts such as debris/third body formation [17,18], tribofilm/transfer-film formation [19-22], grain refinement [23], and subsurface crack formation [24]. Knowledge of the contact geometry is essential for estimating the stresses, which is required to determine interfacial shear strengths as well as values for yield and fracture stresses.

Recent developments in in situ instrumentation have enabled observation of contacting and sliding asperities in real time using transmission electron microscopy (TEM) [3,25-30]. Although standard TEM cannot capture the full 3-D contact geometry, the 2-D image is sufficient to, for example, characterize a PtPt contact accurately enough to correlate it with contact resistance measurements and confirm the presence and character of adsorbates [31], and to determine that a Au-Au contact diffused together in a liquid-like manner when far below the melting point of $\mathrm{Au}$ and further observed transfer of material from one surface to another after contact separation [28]. To date, TEM-based systems could not measure frictional and normal forces at the same time while directly observing the actual contact area at the atomic scale.

Here we present results and analysis of in situ nanoscale Ag-Ag single-asperity experiments, where two isolated Ag protrusions on opposing surfaces are brought into contact via lateral sliding using a customdesigned apparatus housing a nanoelectromechanical systems (NEMS) device. Normal and shear forces are simultaneously resolved. This is a major advance from an initial report of a single $\mathrm{Ag}-\mathrm{Ag}$ nanoasperity sliding junction measurements, where only the lateral force was measured [28]. Here, initial contact, progressive shear, and separation are all observed while monitoring the normal and shear forces, revealing phenomena at play in Ag-Ag contacts never before observed including spontaneous junction formation, 
unstable slip when the asperity tops meet each other, the yield stresses reaching near-ideal strength behavior, and the separation force falling well below predictions used for pure tensile loading.

\section{Results}

Measurement Approach. Figure. 1 shows a schematic of the apparatus, and a sequence from one of six in situ TEM tests conducted of a single nano-asperity nanocontact, extracted from real-time video (examples from five other experiments are shown in Supplementary Figures). The bottom asperity was fixed laterally (it is connected to the cantilever which deflects vertically, in response to contact forces) and the upper asperity was actuated from left to right, as indicated in Fig. 1. The intersection of the plane of contact with the viewing plane gives the contact width. The normal force and shear force reported here are defined as the forces perpendicular and parallel to the plane of contact respectively, at each point of measurement (since the asperity is curved, the orientation of the plane of contact changes as sliding proceeds).

Sliding Experiments. The asperities are initially not in contact (Fig. 1,c i). The load became negative upon of contact (Fig. 1,c ii) due to the van der Waals attraction between the two asperities pulling them into adhesive contact. This is seen as a rapid jump-to-contact instability, consistent with snap-in behavior commonly seen in AFM experiments, and is a result of the attractive force gradient exceeding the normal stiffness of the system, which is primarily determined by the stiffness of the load cantilever. In this experiment, the load increased slightly (became less negative) when the upper asperity climbed over the lower asperity (Fig. 1,c ii -iii), and then decreased (became more negative) when it traveled down the right-side slope of the lower asperity (Fig. 1,c iii-iv). The friction force increased not only as the upper asperity climbed up the bottom asperity (Fig. 1,c ii -iii) but as the upper asperity traveled down the other side of the bottom asperity (Fig. 1,c iv-v). The surfaces then separate (Fig. 1,c vi).

The shape of each asperity prior to contact formation was compared with that after contact separation. Fig. 2a shows the shape of lower asperity before contact, Fig. 2b the shape after separation, and Fig. $2 \mathrm{c}$ a comparison between the two. The apparent volume of the lower asperity increased. To confirm this 
shape change, the same area on the same pair of asperities were scratched to see whether the shape change also occurred with the increase into the actual contact area. The shape of the lower asperity deformed significantly, so either material was added or plastic deformation of the lower asperity occurred (or both). As the volume change is so small, approximately $20 \mathrm{~nm}^{3}$ assuming locally axisymmetric symmetry at each measured height of the asperity, it is not possible to tell which. Sliding experiments using the same procedure were performed for six trials. In all experiments, at most a nanoscale-level amount of deformation and/or transfer was observed (see Supplementary Figures, Figures S.1, S.4, S.7, S.10, and S13).

Extracting Stresses from Force and Contact Width. Figure. 3 shows the forces and stresses at the actual contact interface as a function of the sliding distance. Results for the five other experiments are shown in Supplementary Figures, Figures S.3, S.6, S.9, S.12, and S.15. By recording the forces and contact size concurrently, we are able to calculate the mean normal (tensile in these experiments) and shear stress, measured by dividing the normal or lateral force respectively by the simultaneously obtained contact area, determined by assuming the contact is axisymmetric. We also calculate the von Mises stress (based on the mean stresses at the contact interface) as a function of sliding distance. This is plotted in Fig. 3(d). The von Mises stress is valuable as a measure of the tendency for the onset of plastic flow via the empirical von Mises yield criterion [32]. While it is not particularly useful at stresses beyond the onset of plasticity, it is still directly related to the strain energy density. Thus, we briefly provide a discussion of the evolution of von Mises stress in all experiments. The von Mises stress is calculated as

$$
S_{v M}=\sqrt{\sigma^{2}+3 \tau^{2}}
$$

where the normal stress $\sigma$, and the shear stress $\tau$ are calculated as

$$
\begin{aligned}
& \sigma=\frac{F_{\text {normal }}}{A} \\
& \tau=\frac{F_{\text {shear }}}{A}
\end{aligned}
$$

and the contact area

$$
A=\pi\left(\frac{w}{2}\right)^{2},
$$

where $W$ is the contact width. 
Figure. 3(d) shows that the shear stress is relatively constant throughout the contact event. Because the contact is initially under tensile loading, the tensile normal stress reduces in magnitude as the upper asperity climbs over the lower asperity; this leads to a decrease in the von Mises stress as well. Then, both the tensile normal and the von Mises stress grow substantially in magnitude as the upper asperity descends down the lower asperity. This behavior was seen in 3 of the 6 experiments. In the other 3 , the tensile normal and von Mises stress show monotonic increases with sliding distance. The non-monotonic dependence is attributed to a combination of greater initial tensile normal stress due to an earlier pull-in instability and a more gradual increase of shear stress with sliding distance. The latter factor may be due to the specific arrangement of atoms at the contact interface and the precise misalignment of the two single crystal asperities.

\section{Discussion}

In macroscopic contacts subjected to compressive and tangential loading (even without sliding, i.e. remaining in the static friction regime), ductile materials typically exhibit junction growth (substantial growth in the real contact area, sometimes of over 10 times) [33], due to plasticity induced by the applied shear stress; it can be readily shown from equation (1) that an increase in shear stress must lead to an increase in contact area for the von Mises stress to remain constant [34]. For example, Bowden and Rowe reported a 3-fold increase in the adhesion force between macroscopic silver asperities in vacuum after tangential loading, attributed to junction growth [35]. In stark contrast, junction growth in the present experiments is either not observed, or is very limited (junction growth will still occur even though the normal stresses are tensile, not compressive, in these experiments, since the normal stress shows up quadratically in equation (1)). Fig. 2(c) for example shows a small amount plastic deformation, and similar amounts are seen for the five other experiments as illustrated in the Supplementary Figures, Figures S.2, S.5, S.8, S.11 and S.14. The plastic deformation that is seen is consistently in the form of slight plastic necking near the top of the asperity and oriented in the direction of sliding. This indicates consistently that the level of ductility in these nanoscale 
junctions is substantially less than for macroscopic contacts, and correspondingly, that the yield strength is larger.

In all six experiments, a small instability, in the form of a jump in the sliding distance and a corresponding jump in friction and load forces, is seen at or shortly after point iii (Figures 3, S.3, S.6, S.9, S.12, S.15), i.e. the point where the tips of the two asperities align, i.e., when the asperity interaction transitions from climbing up to climbing down. Further similar instabilities are seen at other points, e.g., between points iv and v in Figure. S.9. Such jumps are similar to yielding events seen in uniaxial tensile tests that are due to plastic events such as dislocation nucleation, dislocation motion, or other more complex events involving (for example, stacking fault-based structures that are found in twinned Ag nanostructures [36] ) and indicate unstable but limited plastic flow. Furthermore, $\mathrm{Ag}-\mathrm{Ag}$ asperities of $3 \mathrm{~nm}$ radii (the same approximate size to those here) were subjected to contact and shear using molecular dynamics recently [37]; they observed very similar instabilities which were directly a result of plastic deformation, supporting our contention that these instabilities are due to yield of the Ag. We note that the instabilities induced at the transition point between climbing up and climbing down may be due to hysteretic unloading behavior, where shrinkage of the contact area as the tensile stress magnitude increases (i.e. the unloading occurring when climbing down) is hindered by interfacial adhesion. In other words, during unloading, tensile elastic strain builds up, then is quickly released in a small strain burst as the contact suddenly shrinks; both elastic and plastic strain release may occur at this point.

Table 1 in the Supplementary Information lists the sliding displacement values and the corresponding stresses at each instability that could be readily identified by an observable, finite slip event. These plastic events occurred at von Mises stresses ranging from 0.58 to $1.78 \mathrm{GPa}$ (mean value \pm standard deviation of $1.23 \pm 0.39)$, which corresponds to an effective shear stress $(1 / \sqrt{3}$ times the von Mises stress) ranging from 0.33 to $1.02 \mathrm{GPa}$ (mean value $0.71 \pm 0.22 \mathrm{GPa}$ ). Considering only the initial yield event for each of the six experiments, the von Mises stresses range from 0.58 to $1.53 \mathrm{GPa}$ (mean value $1.01 \pm 0.35 \mathrm{GPa}$ ), which corresponds to an effective shear stress ranging from 0.33 to $0.88 \mathrm{GPa}$ (mean value $0.58 \pm 0.20 \mathrm{GPa}$ ). These 
values are large. The initial yield occurred at a von Mises stress that is 17-20 times silver's typical bulk yield strength in pure tension of 50-60 MPa (in pure tension, the tensile stress and von Mises stress are identical) $[36,38]$. We compare these to the ideal strength of a crystal in the absence of defects such as dislocations can be roughly estimated to be $\mathrm{G} / 30$ (where $\mathrm{G}=27.8 \mathrm{GPa}$ for silver), i.e., $0.93 \mathrm{GPa}$ [39]. Here, we obtain effective shear stresses at initial yield that reach 35-95\% of this estimated ideal strength of silver. More precisely, the theoretical shear strength of pure Ag with no defects (including dislocations or surfaces) and at $0 \mathrm{~K}$ was calculated from density functional theory by Ogata et al. to be $1.65 \mathrm{GPa}$, based on the $\{111\} /<112>$ slip system [40]. We observe effective shear stresses that reach $20-62 \%$ of this ideal value, despite having proximal surfaces and being measured at $300 \mathrm{~K}$.

Thus, these asperity contacts exhibit strength values under combined loading approaching a significant fraction of the ideal strength. This is surprising given that the asperities were formed after contact and separation via plastic flow and fracture, which one might expect would lead to the nucleation and propagation of a significant number of dislocations. However, plasticity in Ag tends to nucleate in the form of stacking faults (partial dislocation pairs) with a very low stacking fault energy [41] and thus a correspondingly large zone size, making them difficult to nucleate in small volumes. Moreover, that misalignment of the asperities would lead to non-ideal registry of atoms at the interface. As well, the proximity of surfaces, and the temperature being finite, will lead to further reductions of the shear stress at yield from the ideal value of $1.65 \mathrm{GPa}$.

We compare these to experimental measurements of yield of $\mathrm{Ag}$ at the nanoscale. Experimental measurements of the yield of pure Ag nanowires (NW's) in uniaxial tension produced yield strength values of comparable magnitudes to ours. These nanowires are formed with a pentatwinned geometry, but have a low population of defects (other than the five longitudinal twin boundaries). For example, Zhu et al. reported a yield strength of such Ag NW's inside a scanning electron microscope (SEM), with the smallest silver nanowires (34-38 nm diameter) having a yield strength of approximately $2.5 \mathrm{GPa}$ for loading along the $\langle 110\rangle$ direction [42], approaching the ideal strength along the $\langle 110\rangle$ direction of $3.5 \mathrm{GPa}[40,42]$. 
Vlassov et al. [43] performed tensile tests on Ag NW's with diameters from tens to hundreds of nm and obtained a yield strengths ranging from 1-10 GPa, with a median reported value of $4.8 \mathrm{GPa}$. Bernal et al. performed tensile tests of Ag NW's 30 to $120 \mathrm{~nm}$ in diameter, observing yield stress values of approximately $1.6 \mathrm{GPa}$ [44]. Similar measurements by Filleter et al. of Ag NW's 40 to $120 \mathrm{~nm}$ in diameter revealed yield stress values of 2-7 GPa [45]. These TEM results are particularly illuminating as they demonstrate explicitly the near perfection of the NW (such as the absence of dislocations), and that initial yield is correlated with particular plastic events, specifically, surface nucleation of stacking fault decahedrons. The results also showed that initial yield tended to occur at a particular strain value; the corresponding stress value was size-dependent, becoming larger at smaller scales due to a concomitant increase in the elastic modulus with size. MD simulations in those papers provided further support for the findings of high strength being associated with low defect populations at small scales. Leach et al. [46] conducted MD simulations of defect-free silver NW's with varying cross-sectional geometries, finding yield stress varied from 2.5 to 3.5 GPa both for penta-twinned NW's and for untwinned (single crystal) NW's. Masuda and Kizuka [47] performed TEM experiments of Ag-Ag nanoasperity contacts in tensile loading, whose contact widths were controlled down to the scale of 1-2 atoms. The found yield strengths of 0.5-0.6 GPa, and a tensile normal stress at final fracture of approximately $1 \mathrm{GPa}$.

These comparisons illustrate that our observed von Mises stress at yield under combined loading of 0.58 to $1.78 \mathrm{GPa}$ approach or even exceed yield stress values from experiments and simulations in pure tensions of crystalline Ag nanostructures with low defect densities. However, in all but the last example above, our experimental geometry differs substantially as it involves two single crystal asperities brought into contact, where the two crystals may be misaligned, as opposed to the loading of a single, long nanowire. More significantly, the experiments reported here are the first to explore in situ the combined role of tensile normal and shear stresses in the yielding of silver at the nanoscale; few such studies, where both shear and normal forces are resolved, exist for any materials. 
The plastic yielding events mentioned above and indicated in Fig. 3 and the related plots in Supplementary Figures produce lateral slip distances of $0.34-1.58 \mathrm{~nm}$, with an average value of $0.76 \mathrm{~nm}$. The Burgers vector for $\mathrm{Ag}$ is $0.2889 \mathrm{~nm}[48,49]$. Thus, we observe slip distances that are of the order of 1-3 Burgers vectors in size. While this is consistent with the possibility that plastic slip within either or both asperities is the mechanism by which plastic flow is accommodated during these yielding events, we could not in general resolve individual dislocations in the TEM during sliding. Thus, it is also possible the concurrent slip of atomic planes within the asperity occurred, or slip occurred at the interface formed between the two asperities.

We now consider the contact of the two asperities in the context of how polycrystalline metals deform; essentially, the contact is a bicrystal interface with proximal surfaces. While very different compared to an infinite bicrystal or a polycrystalline sample where there is no free volume in the vicinity of the interface, we may extend this analogy by considering that there may be a transition in behavior as a function of asperity size. Namely, as one considers how the mechanics changes as the asperities get smaller, there may initially be an effective Hall-Petch regime where preexisting dislocations become increasingly sparse, leading to a strengthening of the two asperities (dislocation-mediated plasticity). At smaller scales, an effective inverse Hall-Petch regime may occur, where the two-asperity system's strength is reduced. While there are multiple possible mechanisms invoked to explain the inverse Hall-Petch effect in polycrystals, we consider here the mechanism of grain boundary sliding.

Chandross et al. have shown that, at small scales, for potentially arbitrary metallic systems, grain boundary sliding can lead to amorphization of the material at and emanating from the interface [23]. In the Ag deposition process of our experiments, the upper and lower asperities form at a distance from each other and the growth is not epitaxial, which means the crystal orientations of the asperities are not deterministically related. The videos highlight, and Fig. 1 iii (magnified) very clearly show that the apexes are misoriented and suggest that, during sliding, the contact and some distance within each grain may be amorphous. The contact region may thus resemble a low-angle grain boundary, with an amorphous barrier due to the 
low energy barrier of self-mixing of silver. We caution that, without proper exit wave reconstruction, interpretations of white spots as atoms or interatomic voids can be misleading.

That all yield stresses are close to but often somewhat lower than any of the yield stresses in references we have reported here [26-28,40-45,47] suggests that our experiment might be in an effective inverse Hall-Petch regime (again, using polycrystalline materials as an analogy to the asperity system here). The very limited number of dislocations observed also supports this since the inverse Hall-Petch relationship is theorized to originate in the small grains' inability to generate and collect dislocations near grain boundaries. Chandross' amorphization model provides a theoretical upper bound for the maximum shear strength which should occur at or near the transition from grain boundary sliding to dislocation-mediated plasticity. Fig. 4 plots this shear strength along with the experimental effective shear stress values at yield, and the ideal shear strength calculated by Ogata et al. [40]. The fact that the experimental shear stresses at yield fall below the theoretical transition from the inverse Hall-Petch regime to the normal Hall-Petch regime is further evidence that the experiments were in the inverse Hall-Petch regime.

Now we consider the asperity separation process. The experiments had a separation instability at a von Mises stress ranging from 1.28 to $1.81 \mathrm{GPa}(1.50 \pm 0.20)$, which corresponds to an effective shear stress ranging from 0.74 to $1.05 \mathrm{GPa}(0.87 \pm 0.11 \mathrm{GPa})$ (Table 2). These values are also indicative of high intrinsic strength. To put these values in context, we consider the known possibilities for nanoscale contact separation: ductile/atomic necking, uniform separation (ideal cohesive rupture), and crack propagation-like (i.e. JKR-like or flaw-sensitive rupture).

Nanoscale $\mathrm{Au}-\mathrm{Au}$ asperities have been reported to exhibit ductile necking behavior and even extreme, liquid-like behavior during separation; this has been strongly inferred via force and conduction measurements [8,9] and directly observed in TEM studies [28]. Similar behavior has been reported in molecular dynamics simulations [50]. Somewhat similarly, fusing of Ag nanoparticles has been seen in TEM, although the behavior was assisted by the presence of a supporting medium on which Ag atoms could diffuse [51]. Our direct observation using the TEM images with no intervening medium demonstrates that this is not the 
dominant mode of separation; as discussed above, plastic deformation after separation is small, except for one of the experiments as shown in Fig. S9, S12, and S15. As well, a necking instability would normally be manifested as a reduction in the magnitude of the tensile force; this is not seen in any of the experiments. As well, from the force vs. sliding distance plots and from the TEM real-time video, the separation process is observed to involve a sudden instability.

Separation of adhering asperities, even nanoscale in size, is frequently described by adhesive contact mechanics, where the JKR and DMT models provide lower and upper bounds to the pull-off force in pure tensile loading. These models incorporate several assumptions, including homogeneous, isotropic, linear elastic materials; axisymmetric paraboloidal asperities with curvature radii that are large compared to the contact width; and purely normal loading (no shear applied). The normal force at separation (the pulloff force) according to the JKR and DMT models are $\frac{3}{2} \pi R_{e f f} W$ and $2 \pi R_{e f f} W$ respectively, where the effective probe radius $R_{e f f}^{-1}=R_{1}^{-1}+R_{2}^{-1}$ where $R_{l}$ and $R_{2}$ are the radii of the two contacting asperities. These are measured directly from the TEM images before contact and $W$ is the work of cohesion (twice the surface energy) of silver, i.e., $2.52 \mathrm{~J} / \mathrm{m}^{2}[52,53]$. The contact's behavior between the JKR and DMT limit is determined by Tabor's parameter $\mu_{T}=\frac{R_{e f f}^{1 / 3} W^{2 / 3}}{E_{c}^{2 / 3} z_{0}}[54,55]$, where $E_{C}=\frac{E}{2\left(1-v^{2}\right)}$ which for Ag is $48.1 \mathrm{GPa}$ and $z_{0}$ is the equilibrium separation of the surfaces which is taken to also represent the spatial range of the interfacial forces. We assume $z_{0}=0.4 \mathrm{~nm}$, on the order of the lattice spacing of $\mathrm{Ag}$, producing values ranging from $\mu_{T}=0.57-0.81$. Since $\mu_{T}<0.1\left(\mu_{T}>5\right)$ corresponds to the (DMT, JKR) limit, our contacts are in between these limits. The Maugis-Dugdale model allows calculation of the pull-off force for these intermediate cases, given by $\chi \pi R_{\text {eff }} W, 3 / 2<\chi<2$. The values for each experiment are plotted in Fig. 5. The experimental pull-off forces only reach 30-78\% of the MD prediction, showing that this model do not predict the pull-off forces well. However, they do show the same approximately linear increase with $R_{e f f}$. These models treat the separation process as an analog to fracture, with the JKR limit representing the behavior of an ideally brittle Griffith crack. In this "flaw-sensitive" approach, the contact is considered to be an external circular crack under tensile loading that propagates inward at separation. 
Persson has argued that an increase in the separation stress with decreased size will lead to a transition from crack propagation-like separation to a distinct detachment process where bond-breaking occurs uniformly over the entire interface [56]. This will occur at a tensile force given by $\pi w^{2} W / z_{0}$ where $w$ is the contact width at pull-off. The corresponding pull-off forces for Persson's model are also plotted in Fig. 4 , and the results are even further away from the measurements. The contact width below which the transition from crack propagation to ideal cohesive failure (or "flaw-insensitive" decohesion) occurs is given by $8 a^{2} E /\left\{\pi\left(1-v^{2}\right) W\right\}$, or $15.5 \mathrm{~nm}$ in this case. Since our contacts are all smaller than this limit, it is surprising that Persson's model does not appear to apply.

The disagreement with both flaw-sensitive and flaw-insensitive predictions can be resolved by recognizing both sets of predictions do not consider the effect of the shear stress present due to sliding. Shear has been incorporated into adhesive contact mechanics considering mixed-mode loading by Johnson [57] and further supported by Kim, McMeeking, and Johnson for the limit of small contacts where slip tends to occur concurrently (i.e., without annular pre-slip) [58]. Johnson's model predicts that the applied shear stress will reduce the pull-off force, sometimes by appreciable amounts due to the interaction between adhesive and frictional resistance in determining the critical strain energy release rate for fracture. The model relies on an empirical parameter $\alpha$ that represents the degree of interaction between adhesion and friction, with the magnitude of $\alpha$ representing the degree to which shear interacts with adhesion to reduce pull-off forces. Experimental data to determine $\alpha$ are extremely limited. Johnson analyzed prior UHV AFM data [59] for Pt tips in sliding contact with muscovite mica, finding $\alpha=0.2$, corresponding to an $11 \%$ reduction in adhesion induced by sliding [57]. Applying Johnson's model to our data we obtain $\alpha=$ $2.4 \pm 0.8$ (values given in Table 2). This demonstrates a very strong effect of shear on the pull-off force. This result is consistent with the aforementioned MD simulations of Ag-Ag nanoasperities, which reported that the contact area reduced by over $40 \%$ due to sliding, consistent with our measurements. Our results are the first measurement of this parameter using direct in situ observations and indicates that Johnson's model is appropriate for describing these nanoscale Ag asperities. 
It is also possible that the reduced pull-off force is due to the surface energy being lower than that of clean Ag. However, as the experiments are performed in ultrahigh vacuum, the Ag asperities were formed by making and breaking the contact prior to experiments (see Methods), and no contamination was observed on the asperities at the atomic level, contamination cannot explain the reduced pull-off force. As well, nanoscale roughness has been shown to cause large reductions in pull-off forces between diamond and diamondlike carbon asperities [60]. However, this effect occurred for observable levels of roughness in TEM measurements, whereas the asperities here are atomically smooth, to within the resolution of the TEM. We thus expect that nanoscale roughness is not a satisfying explanation for the observed low pull-off force.

Finally, we note that sliding asperities may also adhere strongly and lead to fracture away from the interface, leading to wear and debris formation. Aghababaei et al. [17] calculated a critical contact width for this process to occur in shearing asperities based on the increase in the surface energy produced by fracture and the work done by external forces due to shear stress. If the contact width is smaller than the critical width, asperity fracture and wear debris is occurs. We calculated the critical width for each of the six experiments, and found that the critical contact widths were 170-1900 times larger than the actual contact width measured in the experiment (See Supplementary Discussion 4). Thus, the model of Aghababaei et al. predicts no asperity fracture, consistent with our results.

In summary, we observed $\mathrm{Ag}-\mathrm{Ag}$ nanoasperities in sliding contact using a custom-designed NEMSbased apparatus for in situ TEM observations of contact phenomena. Under combined tensile and shear loading, the von Mises stress at observed initial yield points was approximately 20 times higher than that for macroscopic Ag, and as high as $95 \%$ of the ideal strength of Ag based on an ideal shear strength of G/30. The results indicate the asperities' strength derives from a low population of pre-existing defects, and are consistent with a precited transition from dislocation-mediated plasticity to interfacial amorphization at the sliding interface recently proposed for polycrystalline materials [23]. As well, the asperities separated abruptly with separation forces well below predictions for either ideal cohesive or flaw-sensitive rupture of asperities. The discrepancy can be resolved by accounting for the role of shear stress on adhesive separation, 
as modeled by Johnson [57]. Applying this model to direct observations of contact shows that the coupling effect between shear and adhesion is strong. If so, this implies that shear stress may be able to reduce the contact area and pull-off force of contacting asperities, leading to higher stresses and earlier tensile rupture. This may significantly affect predictions for the contact area, friction coefficient, and degree of plasticity in sliding multi-asperity contacts, topics for which reliable predicative methods do not yet exist.

\section{Acknowledgements}

We gratefully acknowledge Prof. R. A. Bernal, Prof. T. Filleter, Prof. Ju Li, Dr. N. Argibay, Dr. M. Chandross, Dr. J. B. McClimon, Prof. Y. Meng, and Prof. K.-S. Kim for useful discussions. TS acknowledges funding from the Japanese society for the promotion of science and the NSK foundation for advancement of mechatronics. TS, ZM, and RWC acknowledge support from the Air Force Office of Scientific Research under grant FA2386-18-1-4083, and from the National Science Foundation under award CMMI-1761874. 


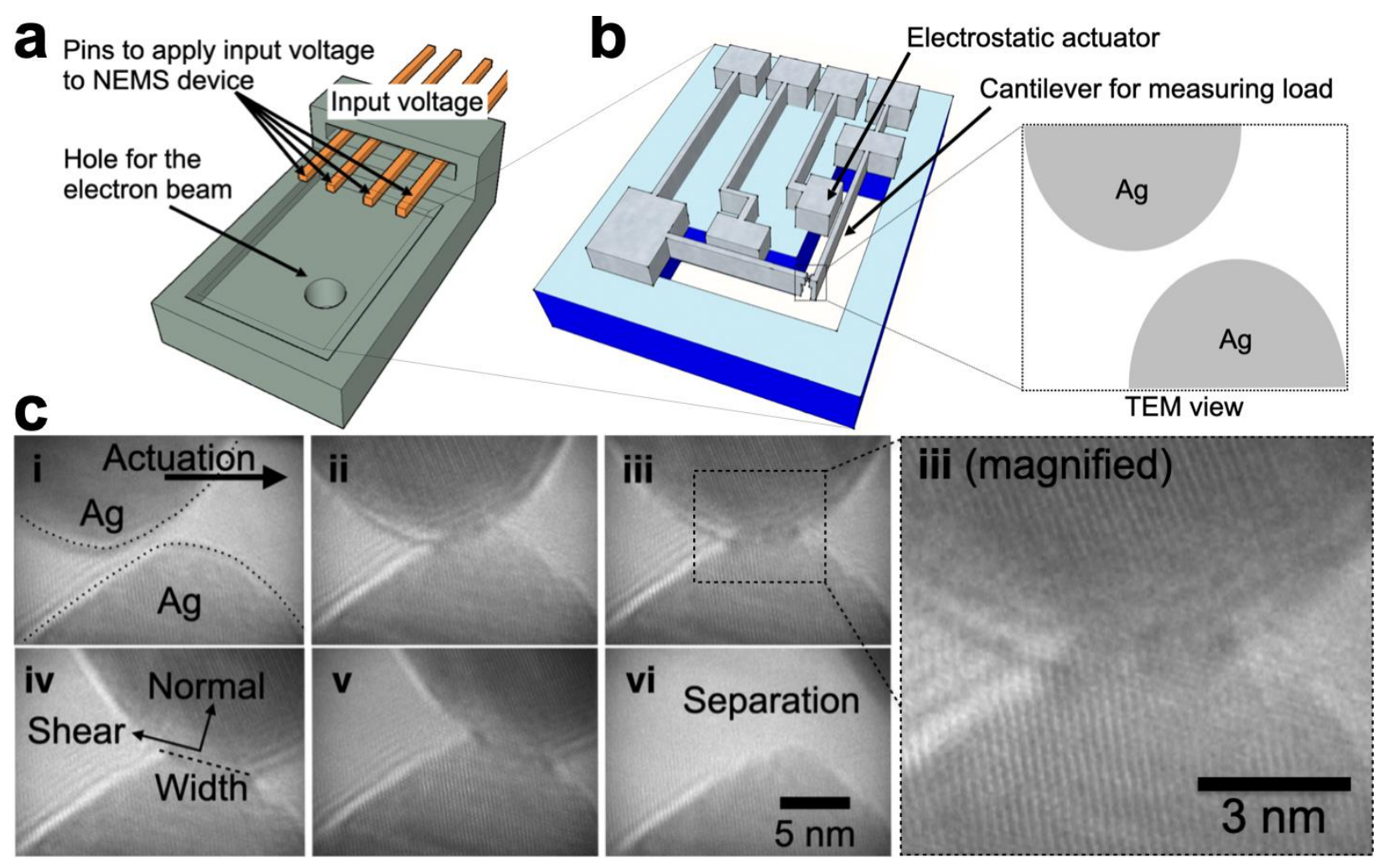

Figure 1 | A custom-designed in situ apparatus is used for nanoscale single asperity friction experiment $[61,62]$. a, Schematic of the stainless steel frame that holds the NEMS device, which is mounted at the sample location of a TEM holder. Four gold wires, used to drive the two electrostatic NEMS actuators, are shown along with a hole for passage of the TEM beam. $\mathbf{b}$, Schematic of the silicon-based NEMS device, showing two orthogonally-oriented cantilevers to measure friction and normal forces, and electrostatic actuators to move asperities in lateral and vertical directions. The inset shows the TEM view of the contact. The upper asperity is the one connected to the cantilever for measuring the friction force. c, Example of a single asperity sliding experiment observed by TEM. i , The upper asperity is actuated in the lateral direction. Initially, the asperities are not in contact. ii, The opposing asperities have come into contact due to attractive forces. iii, iv, $v$, The upper asperity slides laterally across lower asperity. vi, The junction separates. Videos of this experiment are available (See supplementary Movie). 

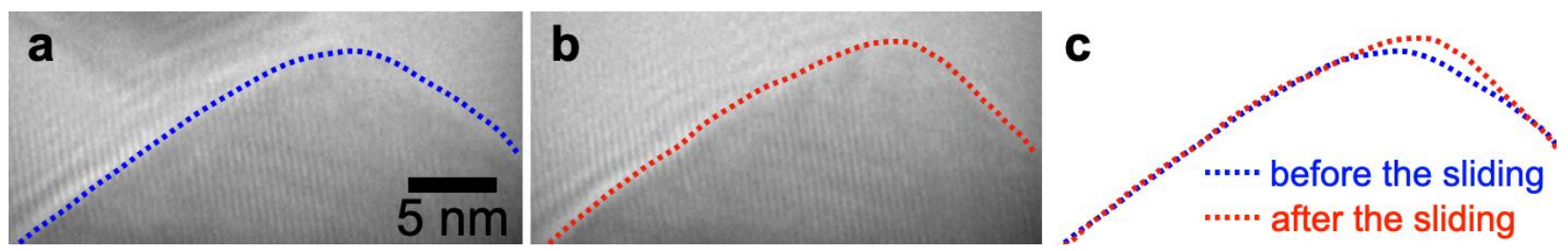

Figure 2 | TEM images demonstrate that nanoscale plastic deformation occurred due to contact and separation. The shape before the contact (a) was compared with the shape after the separation (b).(c) depicts the difference between $\mathrm{i}$ and ii. The lines shown are traced manually while magnifying the image. The plastic displacements are less than $1.0 \mathrm{~nm}$. 


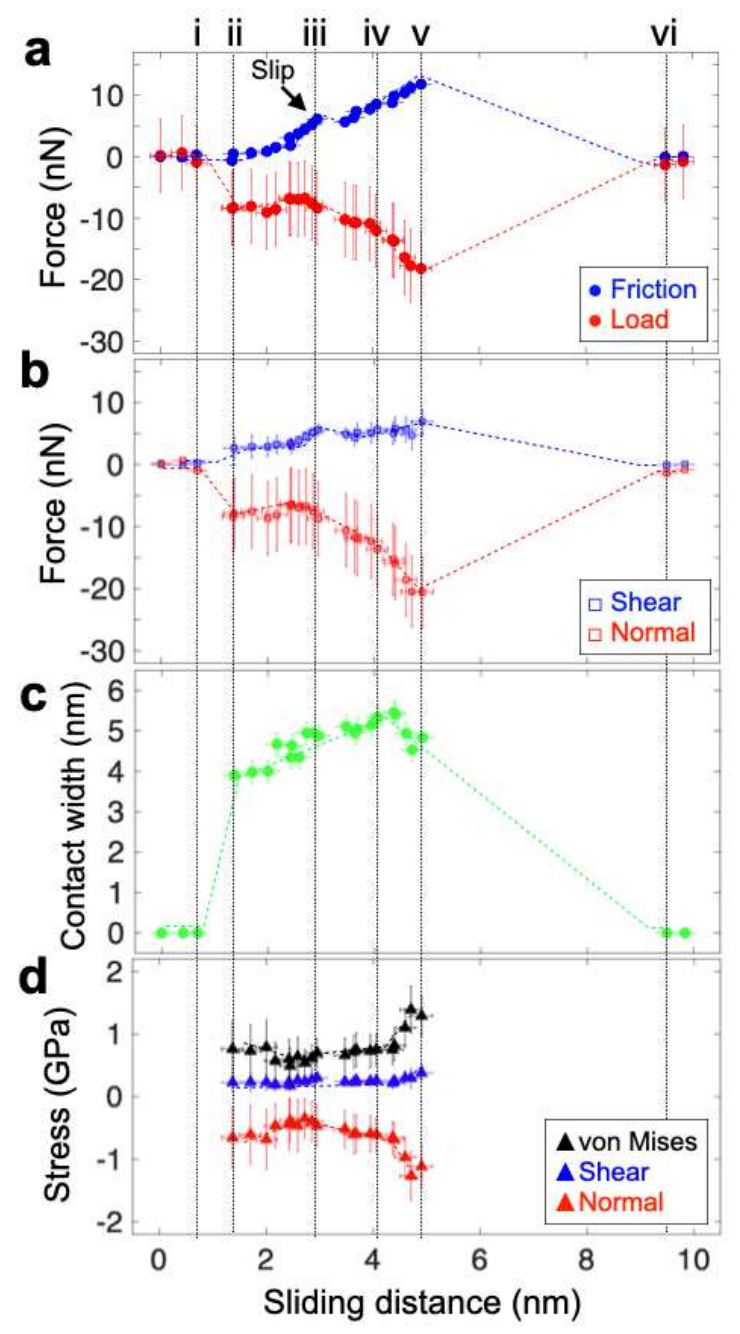

Figure 3 | Forces (from the NEMS device), contact width (from TEM images), and resulting calculated stresses as a function of sliding distance. a, Friction and load forces during an asperity friction measurement. The friction force acts parallel to the direction of the actuation, and the load force acts perpendicular to the friction force, as show in Fig. 2. b, In contrast, the shear force acts parallel to the plane of contact, whose orientation changes during sliding. Similarly, the normal force acts perpendicular to the plane of contact, i.e. perpendicular to the shear force. c, the contact width, measured as the shortest width of the junction. d, The tensile normal stress, shear stress, and von Mises stress, derived from the values of the normal force, the shear force, and the contact width as shown in Eq (1)-(4). The indices i - vi corresponds to the panels in Fig. 1. A video of this experiment is available (See supplementary Movie). 
Table 1 | Shear, normal, von Mises, and effective shear stresses at points for each sliding pass in where unstable slip occurred in the six experiments. The sliding distance at which the slip instability occurred, and the measured slip distance along the lateral direction $X$ and the overall slip distance $R$ (i.e., accounting for lateral and vertical displacement) are also given.

\begin{tabular}{|c|c|c|c|c|c|c|c|c|}
\hline & 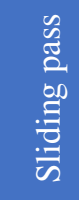 & 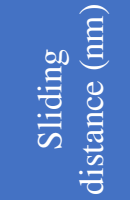 & 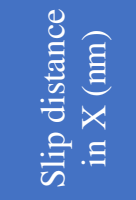 & 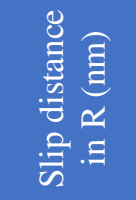 & 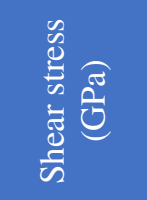 & 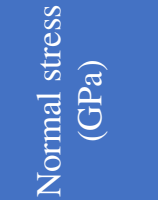 & 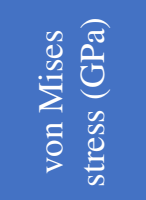 & 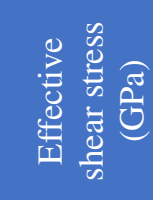 \\
\hline Fig. 3 & $1 \mathrm{st}$ & $2.95 \pm 0.2$ & $0.52 \pm 0.2$ & $0.44 \pm 0.2$ & $0.3 \pm 0.04$ & $-0.47 \pm 0.32$ & $0.70 \pm 0.22$ & $0.40 \pm 0.13$ \\
\hline Fig. S.3 & $1 \mathrm{st}$ & $2.94 \pm 0.2$ & $0.44 \pm 0.2$ & $0.44 \pm 0.2$ & $0.3 \pm 0.06$ & $-0.76 \pm 0.39$ & $0.92 \pm 0.33$ & $0.55 \pm 0.19$ \\
\hline \multirow[t]{2}{*}{ Fig. S.6 } & $1 \mathrm{st}$ & $6.74 \pm 0.4$ & $0.77 \pm 0.4$ & $0.77 \pm 0.4$ & $0.43 \pm 0.17$ & $-1.01 \pm 0.39$ & $1.40 \pm 0.35$ & $0.71 \pm 0.20$ \\
\hline & 2nd & $7.80 \pm 0.4$ & $0.34 \pm 0.4$ & $0.4 \pm 0.4$ & $0.62 \pm 0.18$ & $-1.49 \pm 0.37$ & $1.78 \pm 0.36$ & $0.98 \pm 0.21$ \\
\hline \multirow[t]{3}{*}{ Fig. S.9 } & $1 \mathrm{st}$ & $4.56 \pm 0.4$ & $0.97 \pm 0.4$ & $0.97 \pm 0.4$ & $0.37 \pm 0.04$ & $-0.37 \pm 0.27$ & $0.75 \pm 0.15$ & $0.33 \pm 0.09$ \\
\hline & 2nd & $6.75 \pm 0.4$ & $0.46 \pm 0.4$ & $0.51 \pm 0.4$ & $0.66 \pm 0.1$ & $-0.72 \pm 0.34$ & $1.35 \pm 0.24$ & $0.78 \pm 0.14$ \\
\hline & $3 \mathrm{rd}$ & $7.89 \pm 0.4$ & $0.92 \pm 0.4$ & $0.98 \pm 0.4$ & $0.71 \pm 0.18$ & $-1.29 \pm 0.34$ & $1.78 \pm 0.32$ & $1.02 \pm 0.18$ \\
\hline \multirow[t]{2}{*}{ Fig. S.12 } & $1 \mathrm{st}$ & $11.5 \pm 1.0$ & $1.58 \pm 1.0$ & $1.58 \pm 1.0$ & $0.75 \pm 0.32$ & $-1.09 \pm 0.56$ & $1.70 \pm 0.56$ & $0.88 \pm 0.32$ \\
\hline & 2nd & $14.4 \pm 1.0$ & $0.5 \pm 1.0$ & $0.52 \pm 1.0$ & $0.38 \pm 0.23$ & $-1.36 \pm 0.31$ & $1.51 \pm 0.33$ & $0.87 \pm 0.19$ \\
\hline \multirow[t]{2}{*}{ Fig. S.15 } & $1 \mathrm{st}$ & $13.9 \pm 1.0$ & $1.3 \pm 1.0$ & $1.3 \pm 1.0$ & $0.51 \pm 0.23$ & $-0.68 \pm 0.55$ & $1.12 \pm 0.46$ & $0.62 \pm 0.27$ \\
\hline & 2nd & $15.7 \pm 1.0$ & $0.59 \pm 1.0$ & $0.72 \pm 1.0$ & $0.2 \pm 0.32$ & $-1.06 \pm 0.42$ & $1.13 \pm 0.44$ & $0.65 \pm 0.25$ \\
\hline
\end{tabular}




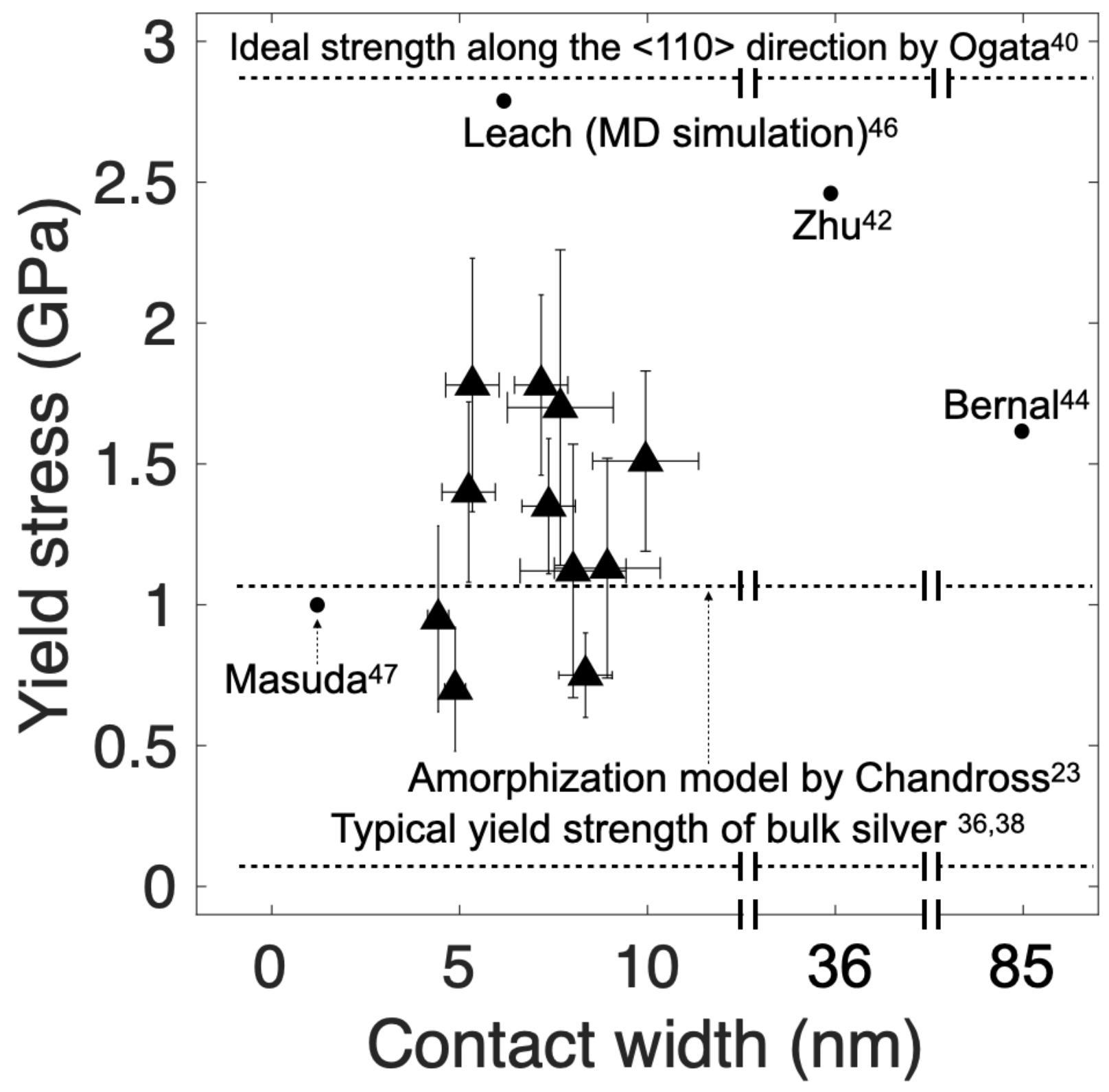

Figure 4 | von Mises stress at slip instabilities. Triangles: values of the von Mises stress measured at observed yield points. Dashed lines and circles show literature values discussed in the text. 
Table 2 | Contact properties at separation. Contact width at separation, asperity radii, Tabor's parameter, experimental pull-off force (force at separation normal to the contact interface), and predicted pull-off forces from the Maugis-Dugdale model (flaw-sensitive rupture) and Persson model (ideal cohesive rupture).

\begin{tabular}{|c|c|c|c|c|c|c|c|c|c|c|}
\hline 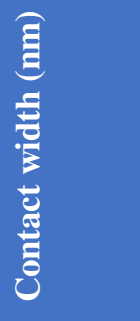 & 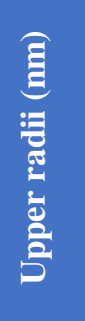 & 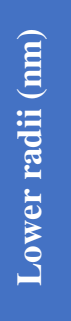 & 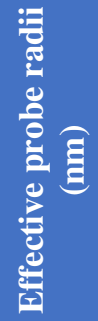 & 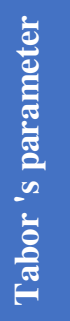 & 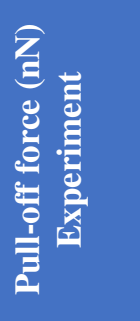 & 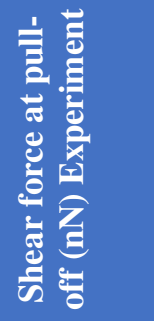 & 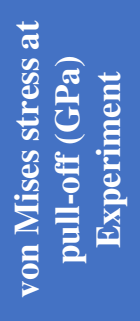 & 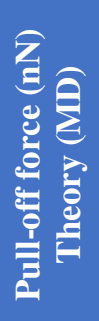 & 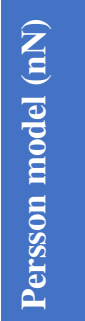 & 8 \\
\hline $4.84 \pm 0.28$ & 12.9 & 6.6 & 4.37 & 0.57 & $20.6 \pm 5.8$ & $6.94 \pm 1.93$ & $1.29 \pm 0.31$ & 57.2 & 116 & 1.93 \\
\hline $3.87 \pm 0.28$ & 12 & 9 & 5.14 & 0.60 & $20.1 \pm 5.5$ & $4.23 \pm 2.61$ & $1.82 \pm 0.46$ & 67.0 & 74.1 & 2.86 \\
\hline $7.99 \pm 0.71$ & 19 & 18.0 & 9.24 & 0.73 & $62.9 \pm 13.4$ & $20.6 \pm 7.76$ & $1.44 \pm 0.27$ & 118.4 & 316 & 1.57 \\
\hline $7.77 \pm 0.71$ & 16.7 & 16.7 & 8.35 & 0.71 & $66.1 \pm 13.0$ & $17.5 \pm 8.46$ & $1.53 \pm 0.28$ & 107 & 299 & 1.56 \\
\hline $9.77 \pm 1.41$ & 26.7 & 22.9 & 12.3 & 0.81 & $122 \pm 21.8$ & $7.7 \pm 21.8$ & $1.63 \pm 0.29$ & 156 & 472 & 3.32 \\
\hline $8.91 \pm 1.41$ & 20.2 & 20 & 10.0 & 0.80 & $80.8 \pm 22.8$ & $8.73 \pm 20.2$ & $1.32 \pm 0.37$ & 127 & 392 & 3.13 \\
\hline
\end{tabular}




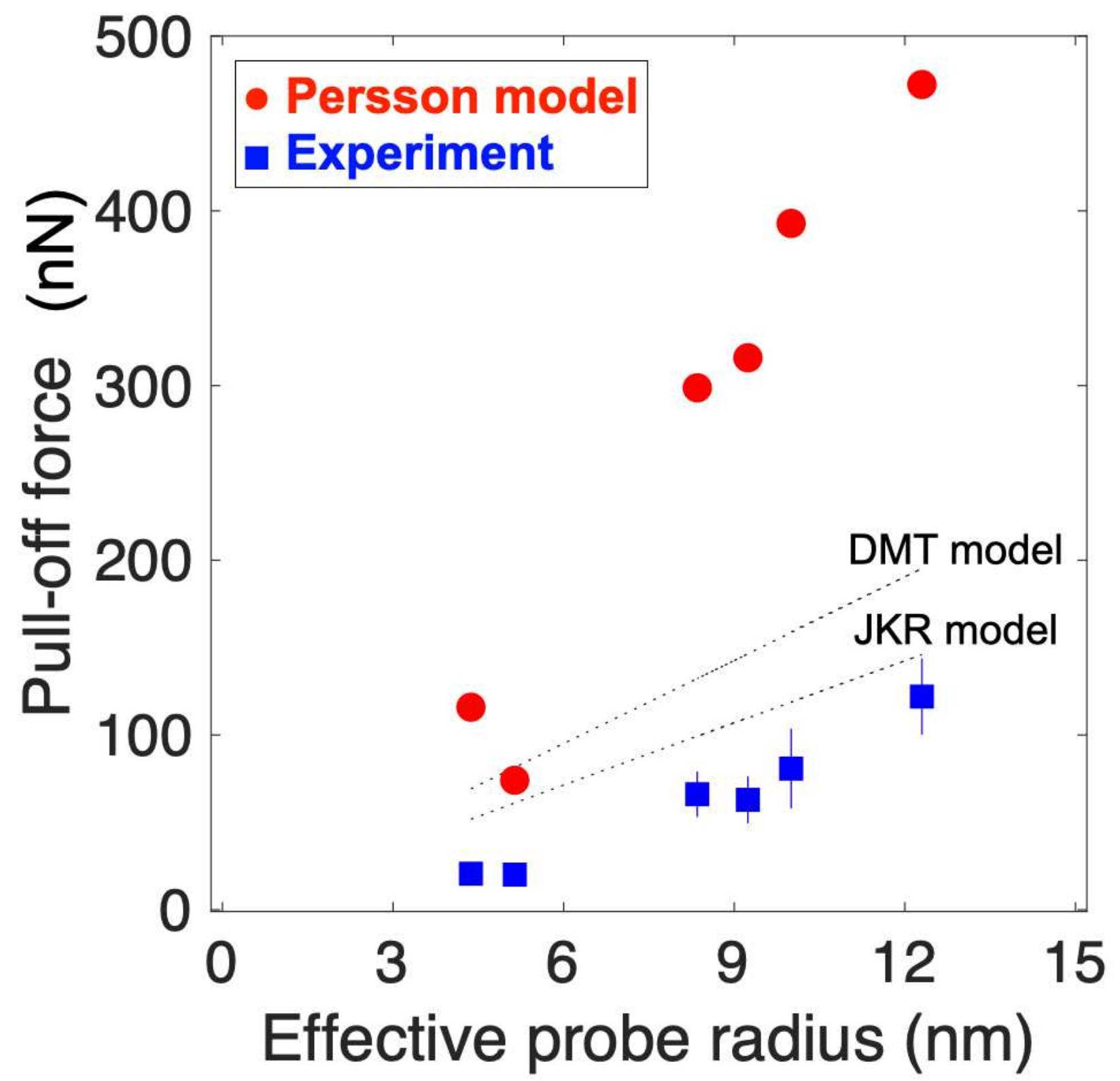

Figure 5 | Measured pull-off force vs. effective probe radius. Blue squares: experiments. Red circles: predicted pull-off force from Persson's model for ideal cohesive rupture. Dashed line: predicted pull-off force from the Maugis-Dugdale model for flaw-sensitive rupture (see Table 2). The experimental pull-off forces are well below all of these predicted values, indicating that another factor, such as the applied shear force, is playing a role in promoting the separation process. 


\section{Methods}

The prototype of a custom-designed NEMS device was first described in reference [61] while the current version is described in reference [62]. The apparatus is illustrated in Fig. 1a,b. The device allows for two displacement axes - in the indentation (load) and the lateral directions - via electrostatically-actuated beams (one for each direction). At the apex of the beams are Ag thin films deposited by thermal evaporation. This produces polycrystalline films with grain sizes of at least 10's of nm. To create a pair of oxide-free asperities, the asperities are brought close together in several ten $\mathrm{nm}$ while applying a pulse voltage between the asperities. The flashing procedure made the surfaces purged and oxide removed. This produces a pair of protruding Ag nanoscale asperities (Fig 1, inset) to examine in the TEM. Each asperity produced in this manner was observed to be crystalline; no grain boundaries were observed within or in the region surrounding the individual asperities. Nominally the two opposing asperities were not cut out of a single crystalline but the roughness formed by the evaporation, could lead to minor misorientation of the crystal axes and other of the two asperities. The device offers the advantages over piezo actuation of increased vibrational and drift stability, linearity, and lack of hysteresis. Forces are calculated by comparing the out-of-contact displacement-actuation voltage response to the in-contact response. The out of contact indentation and lateral displacements are multiplied by the spring constants of the indentation and lateral beams respectively to obtain the force-voltage response. The spring constants of the beams to calculate the forces are obtained experimentally from their resonant frequencies, and the values agree with the analytical and numerical solutions with an accuracy of $1.4 \%$ (See Supplementary Discussion 1). The TEM used was a 200 kV HF2000UHV (Hitachi Ltd., Hitachi-shi, Japan) with a lateral resolution of $0.1 \mathrm{~nm}$ and an ultrahigh vacuum (UHV) chamber pressure of $10^{-8} \mathrm{~Pa}\left(7.5 \times 10^{-11}\right.$ Torr) at room temperature. The UHV conditions greatly reduce the degree of contaminants adsorbed on the surfaces and present in the gas background of the TEM, in contrast to standard TEM which will have several orders of magnitude greater partial pressures of water, molecular hydrogen, hydrocarbons, and other contaminants. Furthermore, a clean Ag surface was created inside the TEM chamber by approaching the probes with a bias to induce field emission. Videos were 
captured with a CCD video camera operating at 2 frames/sec. Due to the high stability of the NEMS actuator, atomic resolution is obtained throughout the majority of frames. The effect of the $200 \mathrm{kV}$ electron beam on the Ag sample was considered. The threshold energy of silver is 5 times higher than the energy due to the electron beam of TEM. Therefore, it cannot displace atomic nuclei to interstitial positions and thereby the beam does not degrade the crystalline perfection of the silver part. The current flowing through the Ag sample was in the range of $\mathrm{pA}$, and the temperature increase due to the electron beam was in the range of $10^{-5} \mathrm{~K}$ (See Supplementary Discussion 2). Therefore, it is reasonable to conclude that electron beam effects are negligibly small.

Experiments consisted of driving the electrostatic actuator to engage lateral motion of the upper asperity with respect to the lower one. This led to contact formation, shearing of the upper asperity across the lower one, and then separation. The contact-shear-separation experiment was performed six times. Throughout the measurement, all the frames were extracted from the TEM video. For each frame, the positions of upper and lower asperities were traced to obtain the relative distances in the lateral and vertical direction. Friction, normal, shear and tensile forces were derived from the lateral and vertical displacement. Stress values such as von-Mises stress were calculated from those force values and the width of the actual contact area measured by real-time video of TEM. The detail of uncertainty analysis was described at Supplementary Discussion 3. 


\section{References}

[1] Sawyer, W. G \& Wahl, K. J. Accessing Inaccessible Interfaces: In Situ Approaches to Materials Tribology. MRS Bulletin 2008, 33 (12), 1145-1150.

[2] Jacobs, T. D. B., Greiner, C., Wahl, K. J. \& Carpick, R. W. Insights into tribology from in situ nanoscale experiments. MRS Bulletin 2019, 44 (06), 478-486.

[3] Lu, Y., Huang, J. Y., Wang, C., Sun, S. \& Lou, J. Cold welding of ultrathin gold nanowires. Nature Nanotechnology, 5, 218-224 (2010).

[4] Pereira, Z. S. \& da Silva, E. Z. Cold welding of gold and silver nanowires: A molecular dynamic study. The Journal of Physical Chemistry C, 115, 22870-22876 (2011).

[5] Szlufarska, I., Chandross, M. \& Carpick, R.W. Recent advances in single-asperity nanotribology. $J$. Phys. D: Appl. Phys. 41, 123001/1-39 (2008).

[6] Mate, C.M. \& Carpick, R.W. Tribology on the small scale: A modern textbook on friction, lubrication, and wear (Oxford University Press, 2019).

[7] Yanson, A.I., Bollinger, G. R., van den Brom, H.E., Agrait, N. \& van Ruitenbeek, J.M. Formation and manipulation of a metallic wire of single gold atoms. Nature, 395, 783-785 (1998).

[8] Rubio, G., Agraït, N. \& Vieira, S. Atomic-sized metallic contacts: Mechanical properties and electronic transport. Phys. Rev. Lett., 76, 2302 (1996).

[9] Agraï, N., Rubio, G. \& Vieira, S. Plastic deformation of nanometer-scale gold connective necks. Phys. Rev. Lett., 74, 3995 (1995).

[10] Pobelov, I. V., Lauritzen, K. P., Yoshida, Y., Jensen, A., Mészáros, G., Jacobsen, K. W., Strange M, Wandlowski, T. \& Solomon, G. C. Dynamic breaking of a single gold bond. Nature Comm., 8, 15931 (2017).

[11] Gosvami, N.N., Filleter, T., Egberts, P. \& Bennewitz, R. Microscopic friction studies on metal surfaces. Tribol. Lett., 39, 19-24 (2010).

[12] Bennewitz, R., Gnecco, E, Gyalog, T. \& Meyer, E. Atomic friction studies on well-defined surfaces. Tribol. Lett., 10, 1 (2001).

[13] Johnson L. K., Kendall, K. \& Roberts, A. D. Surface energy and the contact of elastic solids. Proc. R. Soc. Lond. A, 324, 301-313 (1971).

[14] Derjaguin, B.V., Muller, V. M. \& Toporov, Y. P. Effect of contact deformations on the adhesion of particles, Journal of Colloid and Interface Science, 53(2), 314-326 (1975).

[15] Vishnubhotla, S.B., Chen, R., Khanal, S.R., Hu, X., Martini, A. \& Jacobs, T.D. Matching atomistic simulations and in situ experiments to investigate the mechanics of nanoscale contact. Tribol. Lett., 67, 97 (2019). 
[16] Luan, B. \& Robbins, M.O. The breakdown of continuum models for mechanical contacts. Nature 435, 929-932 (2005).

[17] Aghababaei R., Warner, D. H. \& Molinari, J-F. Critical length scale controls adhesive wear mechanisms, Nature Comm, 7, 11816, (2016).

[18] Godet, M. Third-bodies in tribology. Wear, 136, 29-45 (1990).

[19] Spikes, H. The history and mechanisms of ZDDP. Tribol. Lett., 17, 469-489 (2004).

[20] Erdemir, A., Ramirez, G, Eryilmaz, O. L., Narayanan, B., Liao, Y., Kamath, G. \& Sankaranarayanan, S.K.R.S. Carbon-based tribofilms from lubricating oils. Nature, 536, 67 (2016).

[21] Singer, I. L., Dvorak, S. D., Wahl, K. J. \& Scharf, T. W. Role of third bodies in friction and wear of protective coatings. J. Vac. Sci. Technol. A, 21, 232-240 (2003).

[22] Gosvami, N. N., Bares, J. A., Mangolini, F., Konicek, A. R., Yablon, D. G. \& Carpick, R.W. Mechanisms of antiwear tribofilm growth revealed in situ by single-asperity sliding contacts. Science, 348, 102106 (2015).

[23] Chandross, M., \& Argibay, N. Ultimate strength of metals. Phy. Rev. Lett., 124, 125501 (2020).

[24] Sawyer, W. G., Argibay, N., Burris, D. L. \& Krick, B. A. Mechanistic studies in friction and wear of bulk materials. Ann. Rev. Mat. Res., 44, 395-427 (2014).

[25] Jacobs, T. D. B. \& Carpick, R. W. Nanoscale wear as a stress-assisted chemical reaction. Nature nanotechnology, 8, 108-12 (2013).

[26] Bernal, R. A., Chen, P., Schall, J. D., Harrison, J. A., Jeng, Y-R, \& Carpick, R.W. Influence of chemical bonding on the variability of diamond-like carbon nanoscale adhesion. Carbon, 128, 267-276 (2018).

[27] Sato, T., Ishida, T., Jalabert, L., \& Fujita, H. Real-time transmission electron microscope observation of nanofriction at a single Ag asperity. Nanotechnology, 23, 505701 (2012)

[28] Merkle, A. P. \& Marks, L. D. Liquid-like tribology of gold studied by in situ TEM. Wear, 265, 18641869 (2008).

[29] Liao, Y. \& Marks, L. In Situ Single Asperity Wear at the Nanometre Scale. International Materials Reviews, 62, 99-115 (2017).

[30] Lahouij, I., Dassenoy, F., de Knoop, L., Martin, J.-M. \& Vacher, B. In Situ TEM Observation of the Behavior of an Individual Fullerene-Like MoS2 Nanoparticle in a Dynamic Contact. Tribol. Lett. 42, 133140 (2011).

[31] S. B. Vishnubhotla, Chen, R., Khanal, S. R., Li, J., Stach, E. A., Martini, A. \& Jacobs, T. D. B. Quantitative measurement of contact area and electron transport across platinum nanocontacts for scanning probe microscopy and electrical nanodevices. Nanotechnology, 30, 045705 (2018) 
[32] Beer, F., P., Johnston, E., R., Jr \& DeWolf, J. T. Mechanics of materials. McGraw-Hill, 2002.

[33] Ovcharenko A., Halperin G., \& Etsion I,. In situ and real-time optical investigation of junction growth in spherical elastic-plastic contact. Wear, 264, 1043-50 (2008).

[34] Tabor D. Junction growth in metallic friction: the role of combined stresses and surface contamination. Proc. R. Soc. Lond. A, 251, 378-393 (1959)

[35] Bowden F. P. \& Rowe G. W. The adhesion of clean metals. Proc. R. Soc. Lond. A, 233, 429-442 (1956)

[36] Huo, Y., \& Lee, C.,C., The growth and stress vs. Strain characterization of the silver solid solution phase with indium. Journal of Alloys and Compounds, 661, 372-9 (2016).

[37] Yu, D., Wang, J., Ma, M. and Meng, Y. Effect of Surface Energy on Shearing of Metal Asperities Contact at the Nanoscale. J. Phys. Chem. C 124, 27436-27441 (2020).

[38] Smith, D., R., \& Fickett, F. Low-temperature properties of silver. Journal of research of the National Institute of Standards and Technology, 100, 119 (1995).

[39] Macmillan N. H. The ideal strength of solids, Material Science, p 95-165 (1983)

[40] Ogata, S., Li, J., Hirosaki, N., Shibutani, Y. \& Yip, S. Ideal shear strain of metals and ceramics, Physical Review B, 70, 104104 (2004).

[41] Gallagher, P.C.J. Influence of Alloying, Temperature, and Related Effects on the Stacking Fault Energy. Metallurgical Transactions 1, 2429-2461 (1970).

[42] Zhu, Y., Qin, Q., Xu, F., Ding, Y., Zhang, T., Wiley, B. J. \& Wang, Z., L. Size effects on elasticity, yielding, and fracture of silver nanowires: In situ experiments. Physical Review B, 85, 045443 (2012).

[43] Vlassov, S., Polyakov, B., Dorogin, L. M., Antsov, M., Mets, M., Umalas, M., Saar, R., Lohmus, R., \& Kink, I. Elasticity and yield strength of pentagonal silver nanowires: In situ bending tests. Materials Chemistry and Physics, 143, 3, 1026-1031 (2014).

[44] Bernal, R. A., Aghaei, A., Lee, S., Ryu, S., Sohn, K., Huang. J., Cai, W. \& Espinosa, H. Intrinsic Bauschinger effect and recoverable plasticity in pentatwinned silver nanowires tested in tension, Nano Letters, 15, 139-146 (2015).

[45] Filleter, T., Ryu, S., Kang. K., Yin, J., Bernal, R., A., Sohn, K., Li, S., Huang, J., Cai, W., \& Espinosa, H., D. Nucleation-Controlled Distributed Plasticity in Penta-twinned Silver Nanowires. Small, 8, 19, 2986-2993 (2012)

[46] Leach, A. M., McDowell, M., \& Gall, K. Deformation of top-down and bottom-up silver nanowires, Advanced Functional Materials, 17, 1, 43-53 (2007).

[47] Masuda. H., \& Kizuka. T. Structure, Electrical, and mechanical properties of silver nanocontacts. Jpn. J. Appl. Phys. 49045202 (2010). 
[48] Read, W. T. Jr, Dislocations in Crystals. NewYork: McGraw-Hill (1953).

[49] Cottrell, A. H., Dislocations and Plastic Flow in Crystals. Oxford: Clarendon (1953).

[50] Landman. U., Luedtke, W. D., Burnham. N. A. \& Colton R. J. Atomistic mechanisms and dynamics of adhesion, nanoindentation, and fracture. Science, 248, 454 (1990).

[51] Longo, E.,, Avansi Jr.W., Bettini, J., Andrés, J., \& Gracia, L. In situ Transmission Electron Microscopy observation of Ag nanocrystal evolution by surfactant free electron-driven synthesis, Scientific reports, 6,21498 (2016)

[52] Skriver. H. K., \& Rosengaard, N. M., Surface energy and work function of elemental metals. Phys. Rev. $B, \mathbf{4 6}, 11,1992$

[543] Vitos, L., Ruban A. V., Skriver, H. L. \& Kollar, J. The surface energy of metals. Surface Science, 411, 186-202 (1998)

[54] Maugis, D., Adhesion of spheres: the JKR-DMT transition using a Dugdale model. J. Colloid Interface Sci. 150 (1), 243. (1992)

[55] Grierson, D. S. Flater, E. E. \& Carpick, R. W. Accounting for the JKR-DMT transition in adhesion and friction measurements with atomic force microscopy. J. Adhes. Sci. Technol. 19 (3-5), 291-311 (2005)

[56] Persson, B. Nanoadhesion. Wear, 254 (9), 832-834 (2003).

[57] Johnson, K. L., Continuum mechanics modelling of adhesion and friction. Langmuir. 12 (19), 45104513 (1996)

[58] Kim, K. S., McMeeking, R. M. \& Johnson, K. L. Adhesion, slip, cohesive zones and energy fluxes for elastic spheres in contact. J. Mech. Phys. Sol. 46 (2), 243-66 (1998).

[59] Carpick, R.W., Agraï, N., Ogletree, D.F. and Salmeron, M. Variation of the Interfacial Shear Strength and Adhesion of a Nanometer-Sized Contact. Langmuir 12, 3334-3340 (1996)

[60] Keating, P.,L., Grierson, D.,S., Lefever, J.,A., Turner, K.,T., Harrison, J.,A., \& Carpick, R.,W. The Effect of Atomic-Scale Roughness on the Adhesion of Nanoscale Asperities: A Combined Simulation and Experimental Investigation. Tribol. Lett. 50, 81-93 (2013).

[61] Ishida, T., Nakajima, Y., Kakushima, K., Mita, M., Toshiyoshi, H., \& Fujita, H. Design and fabrication of MEMS-controlled probes for studying the nano-interface under in situ TEM observation. Journal of Micromechanics and Microengineering, 20, 8 (2010).

[62] Sato, T., Tochigi, E., Mizoguchi, T., Ikuhara, Y. \& Fujita, H. An experimental system combined with a micromachine and double-tilt TEM holder. Microelectronic Engineering, 164, 43-47 (2016). 


\section{Supplementary Files}

This is a list of supplementary files associated with this preprint. Click to download.

- SupplimentaryDiscussion14.pdf

- TEMvideo.mov

- SupplimentaryFigures.pdf 